\title{
MKL1 inhibits cell cycle progression through p21 in podocytes
}

Shuang Yang ${ }^{1 *}$, Lingjia Liu' ${ }^{1}$ Pengjuan $\mathrm{Xu}^{2}$ and Zhuo Yang ${ }^{1 *}$

\begin{abstract}
Background: The glomerular podocyte is a highly specialized cell type with the ability to ultrafilter blood and support glomerular capillary pressure. However, little is known about the genetic programs leading to this functionality or the final phenotype.

Results: In the current study, we found that the expression of a myocardin/MKL family member, MKL1, was significantly upregulated during cell cycle arrest induced by a temperature switch in murine podocyte clone 5 (MPC5) cells. Further investigation demonstrated that overexpression of MKL1 led to inhibition of cell proliferation by decreasing the number of cells in S phase of the cell cycle. In contrast, MKL1 knockdown by RNA interference had the opposite effect, highlighting a potential role of MKL1 in blocking G1/S transition of the cell cycle in MPC5 cells. Additionally, using an RT ${ }^{2}$ Profiler PCR Array, p21 was identified as a direct target of MKL1. We further revealed that MKL1 activated p21 transcription by recruitment to the CArG element in its promoter, thus resulting in cell cycle arrest. In addition, the expression of MKL1 is positively correlated with that of p21 in podocytes in postnatal mouse kidney and significantly upregulated during the morphological switch of podocytes from proliferation to differentiation.
\end{abstract}

Conclusions: Our observations demonstrate that MKL1 has physiological roles in the maturation and development of podocytes, and thus its misregulation might lead to glomerular and renal dysfunction.

Keywords: Kidney development, Podocyte, Cell growth arrest, MKL1

\section{Background}

Podocytes, also called visceral glomerular epithelial cells, are terminally differentiated cells overlaying the outer region of the glomerular basement membrane of renal glomeruli. These cells have several key functions including the prevention of proteinuria, synthesis of basement membrane components, regulation of glomerular filtration, and counteraction of intraglomerular hydrostatic pressure [1]. Podocyte injury is typically associated with proteinuria and progressive glomerulosclerosis [2].

Podocytes are derived from epithelial cells originating in the metanephric mesenchyme, which develop into postmitotic terminally differentiated cells, and therefore have similarities to neurons [3,4]. During glomerulogenesis, podocytes proliferate until the S-shape body stage

\footnotetext{
* Correspondence: yangshuang@nankai.edu.cn; zhuoyang@nankai.edu.cn 'Medical School, Tianjin Key Laboratory of Tumor Microenvironment and Neurovascular Regulation, Nankai University, 94 Weijin Road, Tianjin 300071, China

Full list of author information is available at the end of the article
}

and exit the cell cycle at the capillary loop stage $[5,6]$. Podocytes then acquire their fully differentiated phenotype, a process that is not complete until 1 week after birth in the mouse. Mature podocytes tightly regulate and maintain their quiescent and differentiated phenotype, and therefore lost podocytes cannot be replaced by proliferation of neighboring undamaged cells. Indeed, studies have shown that the inability to proliferate contributes to glomerular scarring [7]. Thus, the mechanism responsible for the cell cycle arrest that occurs during nephrogenesis may also participate in maintenance of cell cycle quiescence in mature podocytes.

Megakaryoblastic leukemia 1 (MKL1) was originally found in a study of a chromosomal translocation, t $(1 ; 22)$, which is closely related to the incidence of acute megakaryoblastic leukemia in infants and children [8,9]. MKL1 has been recently shown to be a member of a three-protein family that includes MKL2 and myocardin. These myocardin/MKL proteins serve as serum response factor (SRF) coactivators by binding to SRF and 
strongly activating SRF target genes [10-13]. In contrast to myocardin, which has cardiac and smooth musclespecific expression [10,14-16], MKL1 and MKL2 are expressed in a wide range of embryonic and adult tissues $[8,11,17-20]$. MKL1 regulates many processes including muscle cell differentiation [17], cardiovascular development [18], remodeling of neuronal networks in the developing and adult brain [19], megakaryocytic differentiation and migration [21,22], modulation of cellular motile functions, and epithelial-mesenchymal transition [23-25]. Notably, there is increasing evidence of the involvement of the myocardin/MKL family in suppression of cell proliferation and cell cycle progression. Both myocardin and MKL1 exert anti-proliferative effects in various cell lines [26-28]. Therefore, unraveling the functional pathways in which these proteins have a role and furthering our comprehension of the cellular mechanisms intrinsic to their regulation of cell proliferation will become increasingly important.

In the present study, we found that MKL1 expression was upregulated during temperature-switched growth arrest in murine podocyte clone 5 (MPC5) cells. Overexpression of MKL1 resulted in inhibition of G1/S cell cycle progression in cell viability and EdU cell proliferation assays, whereas MKL1 knockdown had the opposite effect. We further demonstrated that MKL1 induced expression of the cyclin-dependent kinase inhibitor (CKI) p21 during the regulation of cell cycle arrest. Importantly, MKL1 expression was observed in podocytes of the mouse kidney during postnatal development, which was upregulated during the morphological switch of podocytes from proliferation to differentiation.

\section{Results}

\section{Expression of MKL1 is upregulated during} temperature-switched cell growth arrest in MPC5 cells To assess the possible role of myocardin/MKL proteins in podocyte growth arrest, MPC5 cells were respectively maintained at the permissive temperature of $33^{\circ} \mathrm{C}$ and at the nonpermissive temperature of $37^{\circ} \mathrm{C}$ for 10 days. As shown in Figure 1A, a cell viability assay indicated that MPC5 cells cultured at $37^{\circ} \mathrm{C}$ showed a significant decrease in cell number compared with those cultured at $33^{\circ} \mathrm{C}$. The results of immunofluorescence staining in the EdU cell proliferation assay further revealed a marked decrease in the number of cells in $\mathrm{S}$ phase by the temperature switch to $37^{\circ} \mathrm{C}$ (Figure $1 \mathrm{~B}$ and $\mathrm{C}$ ). The percentage of cells in S phase decreased from $46.37 \%$ to $20.03 \%$ as early as 2 days after the temperature switch. At 4-6 days, the percentage of cells in S phase further decreased from $14.97 \%$ to $6.70 \%$, which is consistent with a previous report indicating that the temperature switch induces growth arrest of podocytes in vitro [29].
The expression of myocardin/MKL proteins was then measured during temperature-switched growth arrest in MPC5 cells. qPCR analysis indicated that the temperature switch to $37^{\circ} \mathrm{C}$ induced an approximate 1.8-fold increase in MKL1 mRNA expression compared with the basal level at 2 days (Figure 1D). At 4-10 days, MKL1 expression showed a 2-4-fold increase at the mRNA level. Western blotting was used to confirm the upregulation of MKL1 expression at the protein level (Figure 1E). However, the alteration in myocardin and MKL2 expression was not as evident (Figure 1D). Considering the dominant presence of MKL1 over its other family members, we focused on the effects of MKL1 in subsequent experiments.

\section{MKL1 functions as an effective inducer of cell growth arrest in MPC5 cells}

Next, a mouse MKL1 expression plasmid [11] was transiently transfected into MPC5 cells. Overexpression of MKL1 was assessed by western blotting (Figure 2A). Compared with control cells, the cell viability assay indicated that ectopic expression of MKL1 inhibited MPC5 cell proliferation (Figure 2B). Results of DNA analysis by flow cytometry further confirmed that MKL1overexpressing MPC5 cells had a lower population of $\mathrm{S}$ phase cells and a higher population of G0/G1 phase cells (Additional file 1: Figure S1). The EdU cell proliferation assay revealed a marked decrease in the number of $\mathrm{S}$ phase cells after MKL1 overexpression (Figure 2C). The percentage of cells in $\mathrm{S}$ phase decreased from $55.56 \%$ to $28.39 \%$ at 72 h after transfection of the MKL1 expression plasmid. Furthermore, MPC5 cells were stably transfected with either the MKL1 expression plasmid ( $\triangle$ MKL1) or the empty vector $(\Delta$ Control). Overexpression of MKL1 was then examined by western blotting (Figure 2D). Cell viability and EdU cell proliferation assays confirmed that MKL1 overexpression induced a delay in G1/S phase transition of MPC5 cells (Figure 2E and F).

Therefore, we hypothesized that knockdown of MKL1 by RNA interference would result in an increase in the number of cells in $\mathrm{S}$ phase. To test our hypothesis, a MKL1-targeting shRNA plasmid (shMKL1) or a scrambled control shRNA plasmid (shControl) were transiently transfected into MPC5 cells. Knockdown of MKL1 expression was confirmed by western blotting (Figure 3A). Compared with shControl cells, the cell viability assay indicated that depletion of MKL1 promoted MPC5 cell proliferation (Figure 3B). The results of DNA analysis by flow cytometry further showed that MKL1 knockdown MPC5 cells had a higher population of cells in S phase and a lower population of cells in G0/ G1 phase compared with control cells (Additional file 2: Figure S2). The EdU cell proliferation assay revealed that 


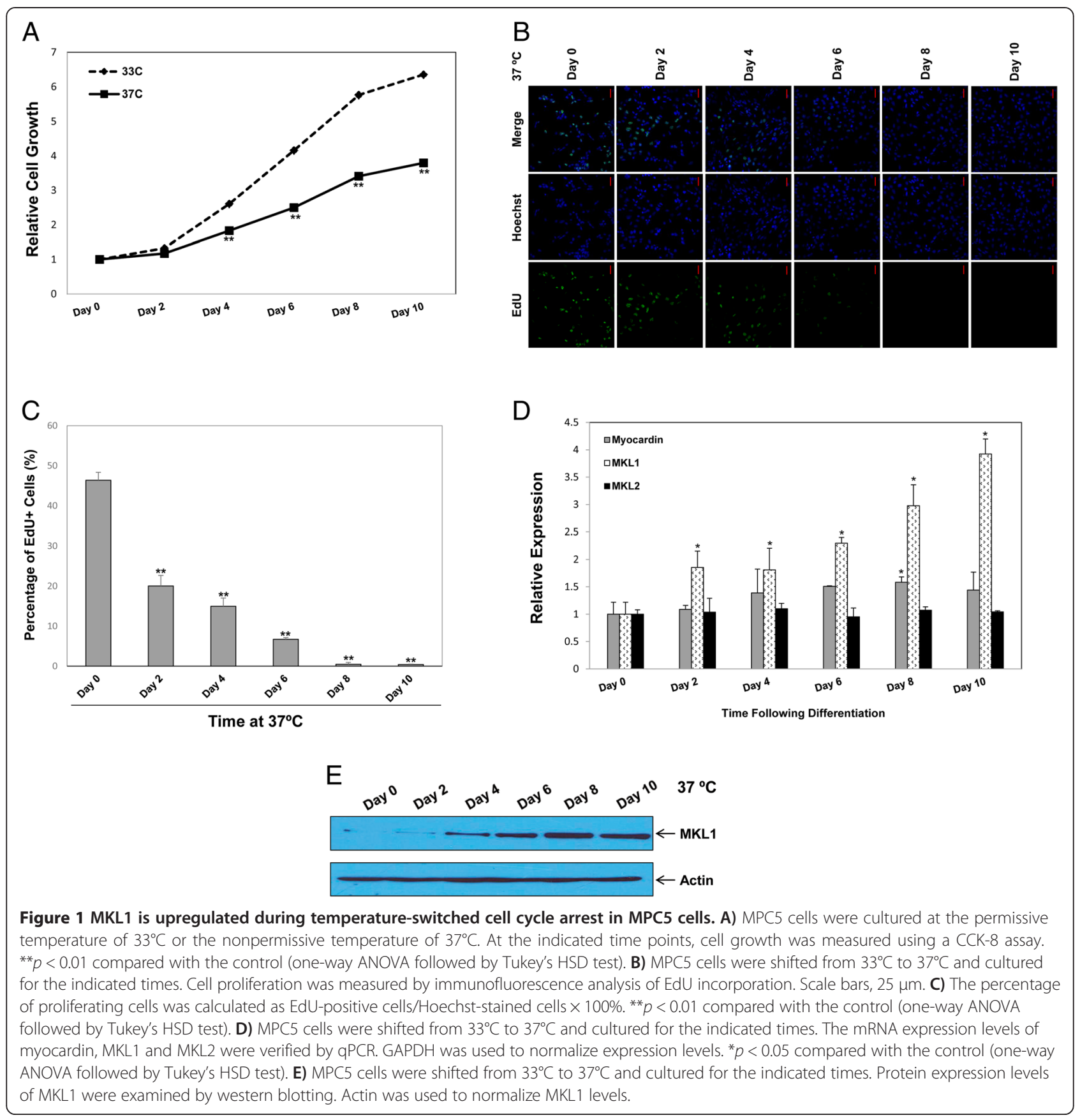

repression of MKL1 resulted in a significant increase in the number of cells in $\mathrm{S}$ phase from $47.40 \%$ to $77.07 \%$ at $72 \mathrm{~h}$ after MKL1 knockdown (Figure 3C). In addition, MPC5 cells were stably transfected with either shMKL1 $(\Delta$ shMKL1) or shControl $(\Delta$ shControl). Knockdown of MKL1 expression was then confirmed by western blotting (Figure 3D). The cell viability and EdU cell proliferation assays confirmed that repression of MKL1 remarkably promoted cell cycle progression through $\mathrm{S}$ phase in MPC5 cells (Figure 3E and F).
MKL1 regulates podocyte proliferation by targeting p21 To identify the potential cellular pathways regulated by MKL1, differences in the mRNA levels of selected signaling molecules were examined using an $\mathrm{RT}^{2}$ Profiler PCR Array by comparing MKL1-expressing MPC5 cells with the control cells. We observed alterations in the expression of several cell cycle regulators, including p21, Gadd45a, Ddit3, E2F2, and cyclin A1 (Table 1). qPCR and western blotting were performed to verify these findings (Figure 4A and Additional file 3: Figure S3). 


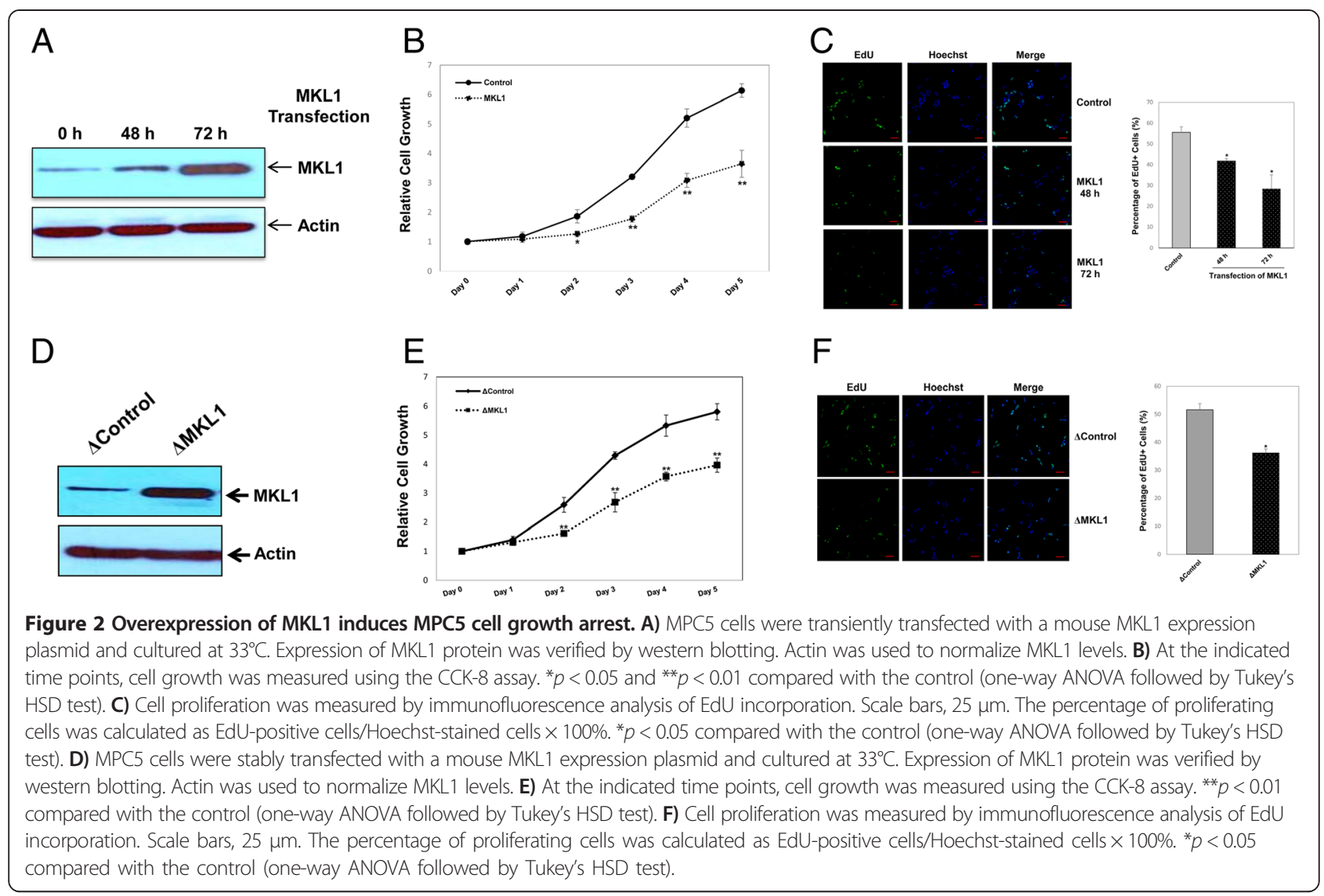

Considering that MKL1 functions with its co-factor SRF by binding to the CArG box in the promoter region of target genes [12,13], we performed a search of the transcription factor database TRANSFAC and identified a CArG box (CCTTTTCTGG) at position -316/-307 in the mouse p21 promoter (Figure 4B). Thus, we assessed whether MKL1 was a bona fide activator of p21 transcription using reporter gene assays. As shown in Figure 4C, MKL1 significantly increased mouse p21 promoter activity of the wild-type $-1562 /+200$ reporter by approximately $49 \%$ relative to the control without MKL1 transfection. Furthermore, we found that MKL1 activated the promoter activity of p21 in a dose-dependent manner (Additional file 4: Figure S4). A series of truncated p21 promoter-reporter constructs were thus generated for analysis, as shown in Figure 4B. The results showed that deletion of the CArG box significantly abolished MKL1-induced transactivation of the p21 promoter compared with that in the control without MKL1 transfection (Figure 4C). Next, we prepared mutants of the CArG box (CCTTTTCTGG to CСТTТТСТTT) by site-directed mutagenesis. We found that mutation of the CArG box was sufficient to interfere with MKL1-activated transcription of the p21 promoter (Figure 4D). Chromatin immunoprecipitation
(ChIP) assays were then performed using an anti-MKL1 antibody, anti-SRF antibody, or control IgG in MPC5 cells. The results indicated that both MKL1 and SRF were able to bind to the p21 promoter during basal conditions in a CArG-dependent manner (Figure 4E). Overexpression of MKL1 resulted in a 1.9-fold increase in its binding to the endogenous p21 promoter in qChIP analysis (Figure 4F). These results suggested that the overexpressed MKL1 in conjunction with SRF promotes p21 transcription by binding to the CArG box in its promoter.

Importantly, to further show that MKL1 regulates podocyte proliferation by targeting p21, MKL1-overexpressing MPC5 cells ( $\triangle$ MLK1) were transfected with a p21targeting shRNA plasmid (shp21). Expression of MKL1 and p21 was assessed by western blotting (Figure 4G). The EdU cell proliferation assay revealed a marked decrease in the number of cells in S phase after MKL1 overexpression, whereas p21 interference remarkably attenuated the MKL1-inhibited cell cycle progression through $\mathrm{S}$ phase. The percentage of cells in $\mathrm{S}$ phase increased from $41.00 \%$ to $67.19 \%$ at $48 \mathrm{~h}$ after transfection of shp21 in MKL1-overexpressing MPC5 cells (Figure $4 \mathrm{H}$ ). These observations confirmed that MKL1 inhibits MPC5 cell proliferation, which is effectively mediated by targeting p21. 


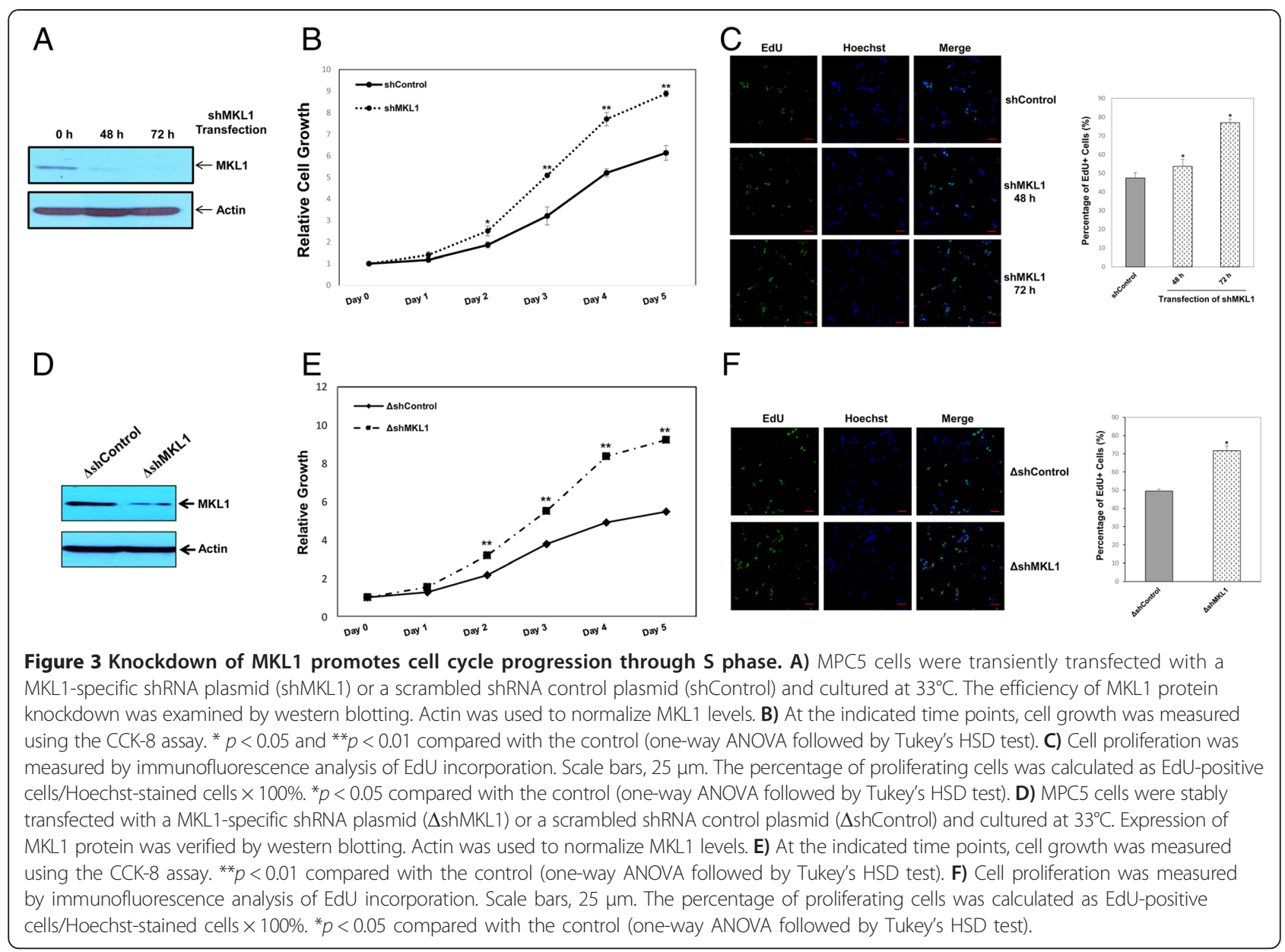

The expression of MKL1 and p21 is positively correlated in podocytes in vivo

Next, we detected the appearance of MKL1 in developing podocytes by examining its expression in the newborn mouse kidney that displays glomeruli at various stages of development from the S-shaped body through the capillary loop stage to mature glomeruli $[5,6]$. Immunofluorescence was used to detect MKL1 expression in the mouse kidney at postnatal day (P) 1, 3, 5, 7, 14, 21, 28 and 49. As seen in Figure 5A b-e, S-shaped and comma-shaped bodies were observed in the renal cortex of immature mice at $\mathrm{P} 1-5$, ultimately vascularizing into a capillary loop of mature nephron at P7. Moreover, MKL1 expression was found at all stages of renal glomerulus and tubule formation during postnatal development (Figure 5A b-i). Importantly, there was an increase in the expression of MKL1 in glomeruli between P5 and P7 (Figure 5A d-e).

Consequently, to address the correlation between MKL1 and p21 expression in newborn mouse kidney, immunofluorescent staining was used to detect p21 expression at P3-28. The results showed a relatively weak expression of p21 in glomeruli at P3-7 during the postnatal development (Figure $5 \mathrm{~B}$ b-d). A remarkable

Table 1 Genes regulated by MKL1

\begin{tabular}{|c|c|c|c|c|c|}
\hline Unigene & GeneBank $^{\mathrm{TM}}$ accession no. & Symbol & Description & $\begin{array}{l}\text { Fold change } \\
\text { (MKL1 vs. Control) }\end{array}$ & $P$ value \\
\hline Mm.195663 & NM_007669 & Cdkn1a & Cyclin-dependent kinase inhibitor 1A (p21) & 4.8402 & 0.0317 \\
\hline Mm.72235 & NM_007836 & Gadd45a & Growth arrest and DNA-damage-inducible 45 alpha & 2.9501 & 0.0268 \\
\hline Mm.110220 & NM_007837 & Ddit3 & DNA-damage inducible transcript 3 & 2.5385 & 0.0282 \\
\hline Mm.307932 & NM_177733 & $\mathrm{E} 2 \mathrm{~F} 2$ & E2F transcription factor 2 & -8.3195 & 0.0146 \\
\hline Mm.4815 & NM_007628 & Ccna1 & Cyclin A1 & -6.1312 & 0.0195 \\
\hline
\end{tabular}




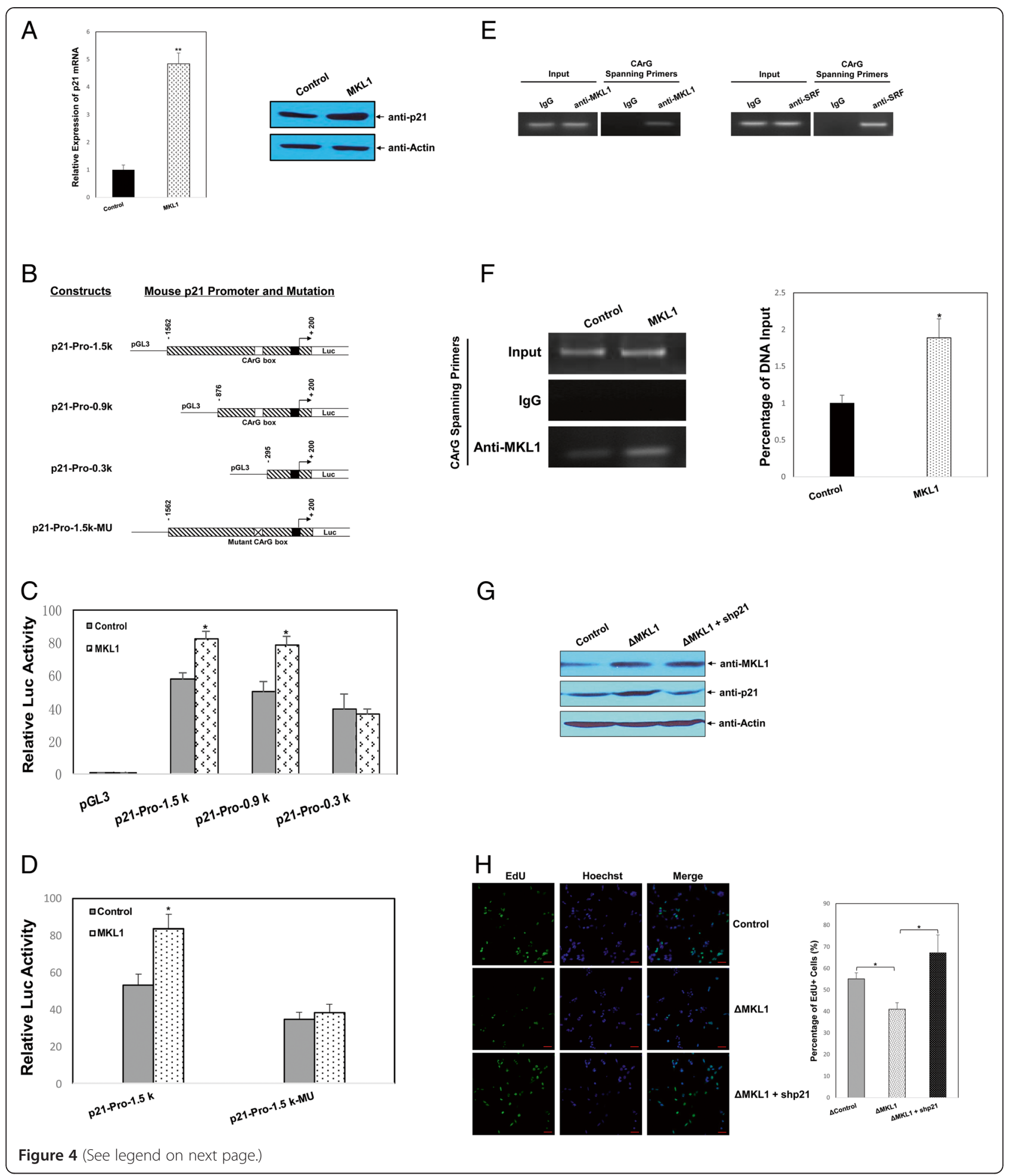


(See figure on previous page.)

Figure 4 MKL1 induces p21 expression. A) The expression of p21 was examined by qPCR and western blotting in MKL1-overexpressing MPC5 cells. GAPDH and actin were used to normalize p21 levels. ${ }^{* *} p<0.01$ compared with the control (unpaired Student's $t$-test). B) Sequential deletions and mutations of the mouse p21 promoter were fused to a luciferase reporter. MPC5 cells in 24-well plates were co-transfected with the MKL1 expression plasmid ( 1 $\mu \mathrm{g} /$ well) and various wild-type $\mathbf{C}$ ) or mutant D) p21 promoter luciferase reporters ( $1 \mu \mathrm{g} /$ well). The luciferase activity of the extracts was determined at $24 \mathrm{~h}$ after transfection using a Betascope analyzer. Luciferase values were normalized to Renilla activities. ${ }^{*} p<0.05$ compared with the empty vector (unpaired Student's t-test). E) ChIP assays were performed using an anti-MKL1 antibody, anti-SRF antibody, or control lgG in MPC5 cells. The association of MKL1 or SRF with the proximal mouse p21 promoter was analyzed by PCR. The amount of input was confirmed by equal loading of chromatin. F) ChIP assays were performed using the anti-MKL1 antibody or control IgG in MKL1-overexpressing MPC5 cells. The association of MKL1 with the proximal mouse p21 promoter was analyzed by PCR or qPCR. The amount of input DNA was confirmed by equal loading of chromatin. ${ }^{*} p<0.05$ compared with the empty vector (unpaired Student's t-test). G) $\Delta$ MKL1 cells were transiently transfected with a p21-specific shRNA plasmid (shp21) and cultured at $33^{\circ} \mathrm{C}$. The efficiency of p21 knockdown was examined by western blotting. Actin was used to normalize MKL1 and p21 levels. H) Cell proliferation was measured by immunofluorescence analysis of EdU incorporation. Scale bars, $25 \mu \mathrm{m}$. The percentage of proliferating cells was calculated as EdU-positive cells/Hoechst-stained cells $\times 100 \%$. ${ }^{*} p<0.05$ compared with the control (one-way ANOVA followed by Tukey's HSD test).

upregulation of p21 expression was then observed between P14 and P21 (Figure 5B e-f), indicating a positive and sequential correlation between MKL1 and p21 expression. Considering that podocytes proliferate until the S-shape body stage and exit the cell cycle at the capillary loop stage during glomerulogenesis, these results are consistent with our notion that MKL1 functions as an effective inducer of cell growth arrest in podocytes, that might be mediated by the regulation of $\mathrm{p} 21$.

\section{Discussion}

Growth arrest and differentiation of podocytes are essential for normal formation of glomeruli in the developing kidney and paramount for normal glomerular function in the mature kidney. The precise cell cycle regulation necessary to establish podocyte quiescence during development has not been fully defined. In the present study, we identified the contribution of one of the myocardin/ MKL proteins, MKL1, to the regulation of MPC5 cell proliferation. During temperature-switched growth arrest of MPC5 cells, MKL1 expression was significantly upregulated above its other family members. Consequently, experiments were performed to assess gain- and loss-of-function of MKL1 to study the effect of MKL1 on MPC5 cell proliferation. We found that overexpression of MKL1 resulted in significant repression of G1/S

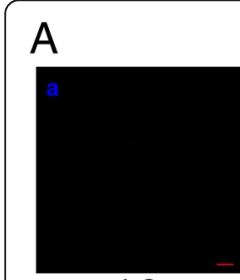

$\lg G$

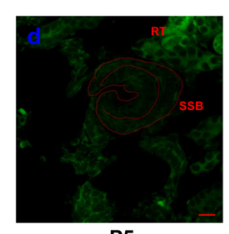

P5

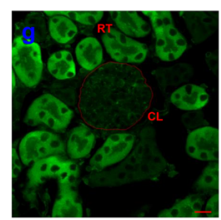

P 21

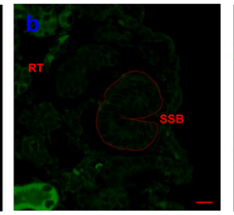

P1

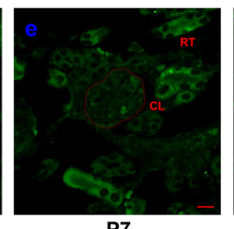

P7

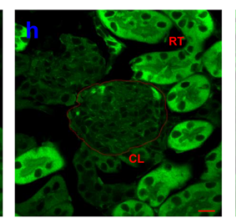

P 28
B

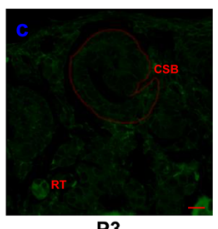

P3
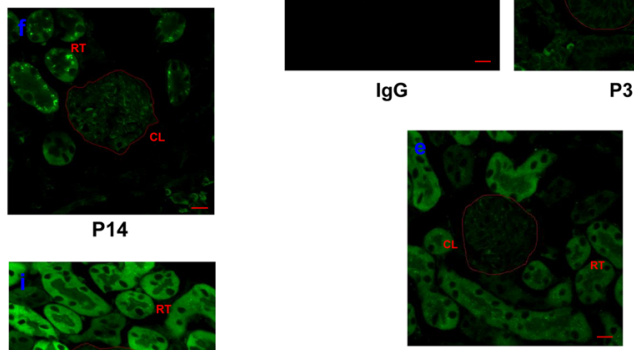

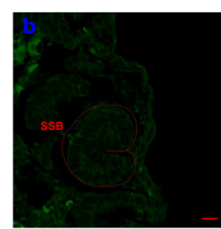

P3

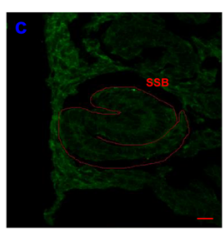

P5

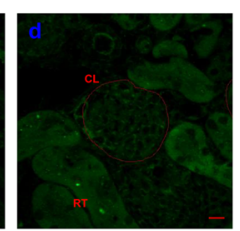

P7
P14
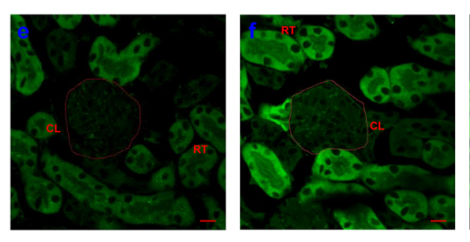

P21

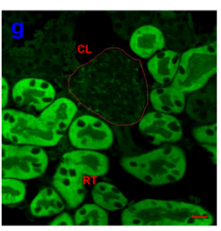

P28

Figure $\mathbf{5}$ The expression of MKL1 and p21 is correlated in podocytes in vivo. A) MKL1 expression was examined by immunofluorescence staining of the mouse renal cortex at P1 (b), P3 (c), P5 (d), P7 (e), P14 (f), P21 (g), P28 (h), and P49 (i). The negative control image shows the renal cortex stained with a species-appropriate lgG (a). Scale bar, $50 \mu \mathrm{m}$. B) The expression of p21 was examined by immunofluorescent staining in the mouse renal cortex at P3 (b), P5 (c), P7 (d), P14 (e), P21 (f) and P28 (g). The negative control image was from the renal cortex, in which the primary antibody was a species-appropriate lgG (a). Scale bar, $50 \mu \mathrm{m}$. The S-shaped body (SSB), comma-shaped body (CSB), capillary loop (CL), and renal tubule (RT) are indicated. 
phase progression of the cell cycle, whereas MKL1 knockdown had the opposite effect. Importantly, we demonstrated that MKL1 activated transcription of the cell cycle inhibitor p 21 by recruitment to its promoter in a CArG element-dependent manner. In addition, MKL1 expression in the mouse kidney during postnatal development showed that MKL1 is expressed in podocytes in vivo, which was significantly upregulated during the morphological switch of podocytes from proliferation to differentiation.

Recent evidence supports an important and complex role of MKL1 in maintenance of proper differentiation in various cell lines including smooth muscle cells [30], myofibroblasts [31], megakaryocytes [22], and adipocytes [32]. Whether MKL1 functions in cell proliferation, however, remains poorly understood. Shaposhnikov et al. reported that MKL1 regulates the expression levels of two proapoptotic Bcl-1 family members, Bok and Noxa, and thus is involved in apoptotic signaling in NIH3T3 fibroblasts [33]. Here, we extended the study to show that elevated expression of MKL1 significantly blocked cell cycle progression by decreasing the number of MPC5 cells in S phase. On the other hand, RNA interference of MKL1 expression resulted in the opposite effect to promote G1/S transition of the cell cycle, confirming a potential role of MKL1 in regulation of cell proliferation. These observations collectively position MKL1 as a potential regulatory factor at the crossroad between cell proliferation and differentiation.

Indeed, myocardin family members have been previously implicated in the regulation of cell proliferation. Myocardin impairs the proliferation of vascular smooth muscle cells, Chinese hamster ovarian $(\mathrm{CHO})$ cells, and leiomyosarcoma cells $[27,28]$. In HT1080 fibrosarcoma cells, myocardin inhibits proliferation at a low cell density and abrogates colony formation in soft agar [34]. However, the suggested molecular mechanisms underlying myocardin/MKL-induced cell growth arrest are conflicting. In human uterine leiomyosarcoma cells, myocardin in conjunction with SRF directly binds to the p21 promoter and induces its expression, thus resulting in G1/S arrest [27]. In contrast, myocardin has been shown to downregulate expression of c-myc, CDK1, CDK2, and S6K, but not p21 and p27, which leads to G2/M arrest and accumulation of polyploidy cells [35]. Our data presented here support the notion that MKL1 functions as an effective regulator to inhibit cell proliferation by altering p 21 expression in MPC5 cells. Significant upregulation of p21 expression at both mRNA and protein levels was observed after transfecting the MKL1 expression plasmid, whereas MKL1 interference resulted in the opposite effect to downregulate p21 expression (Additional file 5: Figure S5), suggesting that a direct mechanism is involved in this regulation. We further demonstrated that MKL1 induced promoter activity of the $p 21$ gene in a dose-dependent manner. Importantly, we found that deletion or mutation of the CArG element in the mouse p 21 promoter remarkably abolished the stimulatory effect on p21 transcription induced by MKL1. Transfection of the MKL1 expression plasmid led to a marked increase in the binding affinity of MKL1 for the endogenous $\mathrm{p} 21$ promoter, indicating a significant role of the CArG element in mediating MKL1-induced expression of $\mathrm{p} 21$. In addition to $\mathrm{p} 21$, we identified obvious candidates involved in MKL1-regulated MPC5 cell proliferation, such as Gadd45a, Ddit3, E2F2, and cyclin A1. However, these genes are not potential targets of myocardin/MKLs/SRF (unpublished data). These results indicate that an SRF-independent mechanism might contribute to MKL-mediated G1/S arrest of the cell cycle.

In the present study, we found that MKL1 was expressed in podocytes of the mouse kidney during postnatal development. Moreover, a significant increase in MKL1 expression was observed between P5 and P7 during postnatal development of the kidney, highlighting a potential role of MKL1 in the physiological and morphological switch of podocytes from proliferation to differentiation. Therefore, using the conditionally immortalized mouse podocyte cell line MPC5, we further revealed that MKL1 functioned as an effective inducer to inhibit cell proliferation and trigger cell cycle arrest at G1/S transition. Several studies have also demonstrated the presence of an intrinsic barrier to replication associated with activation of the cell cycle in podocytes. Re-expression of cell cycle proteins has been reported during glomerular disorders. De novo cyclin A staining is observed in podocytes of children with collapsing glomerulopathy [36] and focal segmental glomerulosclerosis (FSGS) [37]. Positive signals for cyclin D have also been reported in the cellular lesions of FSGS [38]. Recently, strong upregulation of CKIs p21 and p27 was reported in podocytes during Heymann nephritis and in diabetic ZDF-fa/fa rats $[39,40]$. Moreover, the glomerular tufts in crescentic glomerulonephritis strongly express CKIs [41], suggesting that podocytes upregulate CKIs to maintain cell cycle quiescence and preserve normal physiological functions. Here, we extended the study showing that MKL1 acted as an upstream regulator of a variety of cell cycle factors, such as p21 and cyclin A1, to control cell cycle progression in podocytes. In addition, we found significant upregulation of MKL1 expression in the renal tubular cells of newborn mouse kidneys. Recent reports have revealed a potential role of MKL1 in the regulation of renal tubular diseases. For example, Xu et al. reported that MKL1 is induced by glucose and synergizes with glucose to induce collagen expression in cultured renal tubular epithelial cells and the kidneys of mice with diabetic nephropathy, eventually leading to tubulointerstitial 
fibrosis [42]. Moreover, suppressor of cancer cell invasion (SCAI) has been demonstrated to negatively regulate epithelial-mesenchymal transition and renal fibrosis in LLC-PK1 (CL4) proximal tubular epithelial cells, which is at least partially mediated by repression of MKL1 and MKL2 [43]. Taken together, these observations indicate that MKL1 performs physiological roles in maturation and development of the kidney, and thus its dysfunction might lead to glomerular and renal diseases.

\section{Conclusion}

In the present study, we found a potential mechanism of MKL1/p21-mediated cell cycle quiescence in podocytes. Therefore, these findings reveal a novel function of MKL1 during podocyte proliferation and differentiation, furthering our understanding of kidney development and the mechanisms of kidney diseases.

\section{Methods}

\section{Plasmid construction}

The mouse p21 promoter sequence $(-1562 /+200)$ was obtained by PCR from mouse blood genomic DNA and cloned into the pGL3-basic vector (Promega) using the forward primer, 5' -AGCAAGAATTCACAGACCGATG3', and reverse primer, 5'-GTACCTGACACATACA CACC-3'. The mutagenesis of the CArG box in the mouse p21 promoter was performed using the QuickChange Site-Directed Mutagenesis Kit (Stratagene) with the forward primer: 5 -gtactccctgtCCTTTTCTTT gaagtggtgatt-3' and reverse primer: $5^{\prime}$-aatcaccacttc AAAGAAAAGGacaggggagtac- 3 ' .

\section{Cell culture and transfection}

MPC-5 cells were propagated in collagen I-coated dishes at $33^{\circ} \mathrm{C}$ (permissive temperature) in RPMI supplemented with $10 \% \mathrm{FBS}$ and $20 \mathrm{U} / \mathrm{ml}$ of recombinant mouse IFN- $\gamma$ (R\&D). To induce differentiation, the medium was changed to RPMI with 5\% FBS without IFN- $\gamma$, and the cells were shifted to $37^{\circ} \mathrm{C}$ (nonpermissive temperature) for 10 days. Under these conditions, cells underwent growth arrest, increased in size, and developed elongated cell processes. The cells were transfected using TurboFect ${ }^{\mathrm{tm}}$ Transfection Reagent (Fermentas) according to the manufacturer's protocols.

The mouse MKL1 expression plasmid was introduced into MPC5 by transient transfection. G418-resistant clones were isolated over a period of 3-4 weeks. The overexpression of MKL1 was confirmed by western blotting assay.

\section{Cell viability assay}

MPC5 cells transfected with the mouse MKL1 expression plasmid or empty vector were seeded onto 96-well plates at a density of $2 \times 10^{3}$ cells/well and incubated in
RPMI containing 5\% FBS at $33^{\circ} \mathrm{C}$ for 6 days. The cell viability was assessed using the CCK- 8 assay according to the manufacturer's protocols (Dojindo). Six parallel replicates were measured for each sample.

\section{5-ethynyl-2'-deoxyuridine (EdU) cell proliferation assay}

MPC5 cells transfected with the mouse MKL1 expression plasmid or empty vector were seeded onto 24-well plates at 50-60\% confluence. Cells were incubated with $50 \mu \mathrm{M}$ EdU for $2 \mathrm{~h}$ at $48-72 \mathrm{~h}$ after transfection. After the 2-h pulse, the cells were washed twice with PBS and fixed with $4 \%$ paraformaldehyde at room temperature for $30 \mathrm{~min}$. The cells were subsequently washed with glycine $(2 \mathrm{mg} / \mathrm{ml})$ for $5 \mathrm{~min}$, added $0.2 \%$ Trion X-100 for 10 min, washed with PBS twice, and added click reaction buffer (Tris-HCl, pH 8.5, $100 \mathrm{mM}$; CuSO4, $1 \mathrm{mM}$; Apollo 550 fluorescent azide, $100 \mathrm{mM}$; ascorbic acid, $100 \mathrm{mM}$ ) for $30 \mathrm{~min}$ while protecting from light. The cells were then washed again with $0.5 \%$ Triton $\mathrm{X}$ 100 for three times, stained with Hoechst $(5 \mathrm{mg} / \mathrm{ml})$ for $30 \mathrm{~min}$ at room temperature, washed with $0.5 \%$ Triton X-100 for three times. Images were taken and analyzed using Confocal FV1000 (Olympus). EdU positive cells were calculated with (EdU add-in cells/Hoechst stained cells) $\times 100 \%$. At least 200 cells were counted per well.

\section{RNA extraction and quantitative RT-PCR}

Using TRIzol Reagent (Invitrogen), total RNA was extracted from MPC5 cells that were cultured at the nonpermissive temperature of $37^{\circ} \mathrm{C}$ for 10 days. Total RNA $(0.5 \mu \mathrm{g})$ from each sample was used for first-strand cDNA synthesis using M-MLV Reverse Transcriptase (Promega). The specific products of mouse myocardin, MKL1 and MKL2 were amplified by quantitative PCR using the following primers: myocardin, 5'-GATGGG CTCTCTCCAGATCAG-3' (forward) and 5'-GGCTGC ATCATTCTTGTCACTT-3' (reverse); MKL1, 5' -CCCA AAGGTAGCAGACAGTTC-3' (forward) and 5'-GAGT GGGTGATATGGAGGTGG-3' (reverse); and MKL2, 5'-GAGCGAGCCAGAACTGAGAAT-3' (forward) and 5'-ACTCGAATCCACAGGAAGGATG-3' (reverse). The verification of gene expression levels was performed by quantitative RT-PCR using EvaGreen (Biotium). GAPDH was used as an internal control.

\section{Western blotting and antibodies}

Whole cell extract preparation and western blotting with the appropriate antibodies were performed as previously described [44]. The following antibodies (Abs) were used: rabbit polyclonal Ab against MKL1 at 1:800 dilution (ab49311; Abcam), rabbit polyclonal Ab against p21 at 1:500 dilution (10355-1-AP; Proteintech) and mouse monoclonal $\mathrm{Ab}$ against actin at 1:1000 dilution (A-4700; Sigma). 


\section{$\mathrm{RT}^{2}$ Profiler $^{\mathrm{TM}} \mathrm{PCR}$ array}

Total RNA was extracted from MPC5 cells stably transfected with the mouse MKL1 expression plasmid $(\triangle \mathrm{MKL} 1)$ or empty vector ( $\Delta$ Control). For PCR array experiments, an $\mathrm{RT}^{2}$ Profiler custom PCR array was used to simultaneously examine the mRNA levels of 84 genes closely associated with cell cycle, including 5 "housekeeping genes", in 96-well plates following the manufacturer's protocol (PAMM-020Z, QIAGEN). Briefly, first-strand cDNAs were synthesized from $1 \mu \mathrm{g}$ of total RNA using the TaqMan RT reagent kit (QIAGEN) according to the manufacturer's protocol. The reaction mixtures $(25 \mu \mathrm{l})$ were incubated at $25^{\circ} \mathrm{C}$ for $10 \mathrm{~min}$, followed by incubation at $48^{\circ} \mathrm{C}$ for $30 \mathrm{~min}$ and $95^{\circ} \mathrm{C}$ for $5 \mathrm{~min}$, then cooled on ice. Arrays were performed independently at least three times for each cell line; values were obtained for the threshold cycle $(\mathrm{Ct})$ for each gene and normalized using the average of five housekeeping genes on the same array (Actb, B2m, Gapdh, Gusb, and Hsp90ab1). The Ct values for housekeeping genes and a dilution series of ACTB were monitored for consistency between the arrays. The change ( $\Delta \mathrm{Ct})$ between $\Delta \mathrm{MKL} 1$ and $\Delta$ Control was determined using the formula $\Delta \mathrm{Ct}=\mathrm{Ct}(\Delta \mathrm{MKL} 1)-\mathrm{Ct}$ ( $\Delta$ Control), and the fold change was determined using the formula fold change $=2^{(-\Delta C t)}$. The resulting values were reported as fold change; only genes showing twofold or greater change were considered. The negative controls ensured the absence of DNA contamination and set the threshold for determining absence versus presence of expression.

\section{Luciferase assay}

MPC5 cells were co-transfected with the wild-type or mutant mouse p21 promoters and MKL1 expression plasmid in 24-well plates. Lysates were prepared at $24 \mathrm{~h}$ after transfection, and luciferase activities were measured using the Dual-Luciferase Reporter Assay System (Promega) according to the manufacturer's protocols. The luciferase activities were normalized to the values for Renilla luciferase.

\section{Chromatin immunoprecipitation (CHIP)}

ChIP assays were performed using reagents commercially obtained from Upstate, essentially according to the manufacturer's instructions. The antibodies used in these experiments were rabbit polyclonal Ab against MKL1 (ab49311; Abcam) and anti-rabbit normal IgG (sc-2345, Santa Cruz). The amounts of each specific DNA fragment in immunoprecipitates were determined by PCR or quantitative PCR reactions. The fragment of mouse p21 promoter, containing the CArG box, was amplified using the forward primer: 5 '-CCCTCGTGCTTA GACCA-3', and reverse primer: 5'-GCTGTTGCTGC TACCCA-3'.

\section{Immunofluorescent analysis}

Tissue samples were placed in $4 \%$ paraformaldehyde in PBS over 2 hours at $4^{\circ} \mathrm{C}$, immersed in $30 \%$ sucrose overnight at $4^{\circ} \mathrm{C}$, and then were embedded in OCT compound (Tissue-Tek, Miles) and sectioned at $5 \mathrm{~mm}$ (Leica CM 1850, Leica Instruments) for the morphological and immunohistochemistry study. Sections were washed three times in PBS for five minutes each and incubated in blocking buffer with $10 \%$ serum of the secondary antibody host species for 1 hour at room temperature. After that, sections were incubated with rabbit polyclonal $\mathrm{Ab}$ against MKL1 (ab49311; Abcam) and p21 (10355-1-AP; Proteintech) at 1:100 dilution overnight at $4{ }^{\circ} \mathrm{C}$. After being washed three times for 10 minutes with PBS at room temperature, sections were incubated with Alexa 488-conjugated anti-rabbit IgG for 3 hours at room temperature. Negative control samples were treated with species-appropriate IgG instead of primary antibody. Images were taken and analyzed using Confocal FV1000 (Olympus).

\section{Statistical analysis}

SPSS 17.0 software (SPSS) was used for statistical analysis. The data from all the experiments are presented as means $\pm \mathrm{SD}$ and represent three independent experiments. One-way analysis of variance (ANOVA) was used to compare means between treatment groups and Tukey's HSD (honestly significant difference) test was used to evaluate the statistically significant differences between groups. Where appropriate, Student's $t$-test for unpaired observations was applied. A $p$-value $<0.05$ was considered significant.

\section{Additional files}

Additional file 1: Figure S1. Overexpression of MKL1 induces cell cycle delay at the G1-S phase transition.

Additional file 2: Figure S2. Knockdown of MKL1 promotes cell cycle progression at the G1-S phase transition.

Additional file 3: Figure S3. MKL1 inhibits cell proliferation by altering the levels of cell cycle regulators.

Additional file 4: Figure S4. MKL1 activates the transcriptional activity of p21 in a dose-dependent manner.

Additional file 5: Figure S5. Knockdown of MKL1 results in upregulation of p21 expression.

Competing interests

The authors declare that no competing interests exist.

Authors' contributions

Contributed reagents/materials/analysis tools: SY ZY. Wrote the paper: SY. Conceived and designed the experiments (Figures 1, 2, 3 and 4 and supporting materials): SY LL ZY. Conceived and designed the experiments (Figure 5): PJX ZY. Acquisition of data (Figures 1, 2, 3 and 4 and supporting materials): SY LUL. Acquisition of data (Figure 5): PJX. All authors read and approved the final manuscript. 


\section{Acknowledgements}

This work was partly supported by grant from the National Basic Research Program of China (2011CB944003), the China National Nature Science Foundation (No. 81072153, No. 81272184), the New Century Excellent Talents Supporting Program (No. NCET-11-0260), and the Tianjin Natural Science Foundation (No. 13JCZDJC30200). The mouse MKL1 expression plasmid is a kind gift from Dr. Da-Zhi Wang, Children's Hospital Boston.

\section{Author details}

${ }^{1}$ Medical School, Tianjin Key Laboratory of Tumor Microenvironment and Neurovascular Regulation, Nankai University, 94 Weijin Road, Tianjin 300071, China. ${ }^{2}$ Tianjin University of Traditional Chinese Medicine, Tianjin 300193, China.

Received: 7 August 2014 Accepted: 16 January 2015 Published online: 12 February 2015

\section{References}

1. Pavenstädt $\mathrm{H}$, Kriz W, Kretzler M. Cell biology of the glomerular podocyte. Physiol Rev. 2003;83(1):253-307.

2. Greka A, Mundel P. Cell biology and pathology of podocytes. Annu Rev Physiol. 2012;74:299-323.

3. Kreidberg JA. Podocyte differentiation and glomerulogenesis. J Am Soc Nephrol. 2003;14(3):806-14

4. Kobayashi N, Gao SY, Chen J, Saito K, Miyawaki K, Li CY, et al. Process formation of the renal glomerular podocyte: is there common molecular machinery for processes of podocytes and neurons? Anat Sci Int. 2004;79(1):1-10.

5. Marshall CB, Shankland SJ. Cell cycle regulatory proteins in podocyte health and disease. Nephron Exp Nephrol. 2007;106(2):e51-9.

6. Price PM. A role for novel cell-cycle proteins in podocyte biology. Kidney Int. 2010;77(8):660-1

7. Kriz W, Shirato I, Nagata M, LeHir M, Lemley KV. The podocyte's response to stress: the enigma of foot process effacement. Am J Physiol Renal Physiol. 2013;304(4):F333-47.

8. Ma Z, Morris SW, Valentine V, Li M, Herbrick JA, Cui X, et al. Fusion of two novel genes, RBM15 and MKL1, in the $t(1 ; 22)(p 13 ; q 13)$ of acute megakaryoblastic leukemia. Nat Genet. 2001;28(3):220-1.

9. Mercher T, Coniat MB, Monni R, Mauchauffe M, Nguyen Khac F, Gressin L, et al. Involvement of a human gene related to the Drosophila spen gene in the recurrent $t(1 ; 22)$ translocation of acutemegakaryocytic leukemia. Proc Natl Acad Sci U S A. 2001;98(10):5776-9.

10. Wang D, Chang PS, Wang Z, Sutherland L, Richardson JA, Small E, et al. Activation of cardiac gene expression by myocardin, a transcriptional cofactor for serum response factor. Cell. 2001;105(7):851-62.

11. Wang DZ, Li S, Hockemeyer D, Sutherland L, Wang Z, Schratt G, et al. Potentiation of serum response factor activity by a family of myocardinrelated transcription factors. Proc Natl Acad Sci U S A. 2002;99(23):14855-60.

12. Cen B, Selvaraj A, Burgess RC, Hitzler JK, Ma Z, Morris SW, et al. Megakaryoblastic leukemia 1, a potent transcriptional coactivator for serum response factor (SRF), is required forserum induction of SRF target genes. Mol Cell Biol. 2003;23(18):6597-608.

13. Selvaraj A, Prywes R. Expression profiling of serum inducible genes identifies a subset of SRF target genes that are MKL dependent. BMC Mol Biol. 2004:5:13.

14. Wang Z, Wang DZ, Pipes GC, Olson EN. Myocardin is a master regulator of smooth muscle gene expression. Proc Natl Acad Sci U S A. 2003;100(12):7129-34.

15. Yoshida T, Sinha S, Dandré F, Wamhoff BR, Hoofnagle MH, Kremer BE, et al. Myocardin is a key regulator of CArG-dependent transcription of multiple smooth muscle marker genes. Circ Res. 2003;92(8):856-64.

16. Chen J, Kitchen CM, Streb JW, Miano JM. Myocardin: a component of a molecular switch for smooth muscle differentiation. J Mol Cell Cardiol. 2002;34(10):1345-56.

17. Selvaraj A, Prywes R. Megakaryoblastic leukemia-1/2, a transcriptional co-activator of serum response factor, is required for skeletal myogenic differentiation. J Biol Chem. 2003;278(43):41977-87.

18. Parmacek MS. Myocardin-related transcription factors: critical coactivators regulating cardiovascular development and adaptation. Circ Res. 2007;100(5):633-44
19. Mokalled MH, Johnson A, Kim Y, Oh J, Olson EN. Myocardin-related transcription factors regulate the C $\mathrm{dk} 5 /$ Pctaire 1 kinase cascade to control neurite outgrowth, neuronal migration and brain development. Development. 2010;137(14):2365-74

20. Ly DL, Waheed F, Lodyga M, Speight P, Masszi A, Nakano H, et al. Hyperosmotic stress regulates the distribution and stability of myocardinrelated transcription factor, a key modulator of the cytoskeleton. Am J Physiol Cell Physiol. 2013;304(2):C115-27.

21. Cheng EC, Luo Q, Bruscia EM, Renda MJ, Troy JA, Massaro SA, et al. Role for MKL1 in megakaryocytic maturation. Blood. 2009;113(12):2826-34.

22. Smith EC, Teixeira AM, Chen RC, Wang L, Gao Y, Hahn KL, et al. Induction of megakaryocyte differentiation drives nuclear accumulation and transcriptional function of MKL1 via actin polymerization and RhoA activation. Blood. 2013;121(7):1094-101.

23. Fan L, Sebe A, Péterfi Z, Masszi A, Thirone AC, Rotstein OD, et al. Cell contact-dependent regulation of epithelial-myofibroblast transition via the rho-rho kinase-phospho-myosin pathway. Mol Biol Cell. 2007;18(3):1083-97.

24. Elberg G, Chen L, Elberg D, Chan MD, Logan CJ, Turman MA. MKL1 mediates TGF-beta1-induced alpha-smooth muscle actin expression in human renal epithelial cells. Am J Physiol Renal Physiol. 2008;294(5):F1116-28.

25. Mihira H, Suzuki HI, Akatsu Y, Yoshimatsu Y, Igarashi T, Miyazono K, et al. TGF- $\beta$-induced mesenchymal transition of MS-1 endothelial cells requires Smad-dependent cooperative activation of Rho signals and MRTF-A. J Biochem. 2012;151(2):145-56.

26. Descot A, Hoffmann R, Shaposhnikov D, Reschke M, Ullrich A, Posern G. Negative regulation of the EGFR-MAPK cascade by actin-MAL-mediated Mig6/Errfi-1 induction. Mol Cell. 2009;35(3):291-304.

27. Kimura Y, Morita T, Hayashi K, Miki T, Sobue K. Myocardin functions as an effective inducer of growth arrest and differentiation in human uterine leiomyosarcoma cells. Cancer Res. 2010;70(2):501-11.

28. Tang $\mathrm{RH}$, Zheng $\mathrm{XL}$, Callis TE, Stansfield WE, He J, Baldwin AS, et al. Myocardin inhibits cellular proliferation by inhibiting NF-kappaB(p65)-dependent cell cycle progression. Proc Natl Acad Sci U S A. 2008;105(9):3362-7.

29. Saleem MA, O'Hare MJ, Reiser J, Coward RJ, Inward CD, Farren T, et al. A conditionally immortalized human podocyte cell line demonstrating nephrin and podocin expression. J Am Soc Nephrol. 2002;13(3):630-8.

30. Jeon ES, Park WS, Lee MJ, Kim YM, Han J, Kim JH. A Rho kinase/myocardinrelated transcription factor-A-dependent mechanism underlies the sphingosylphosphorylcholine-induced differentiation of mesenchymal stem cells into contractile smooth musclecells. Circ Res. 2008;103(6):635-42.

31. Velasquez LS, Sutherland LB, Liu Z, Grinnell F, Kamm KE, Schneider JW, et al. Activation of MRTF-A-dependent gene expression with a small molecule promotes myofibroblast differentiationand wound healing. Proc Natl Acad Sci U S A. 2013;110(42):16850-5

32. Nobusue $H$, Onishi $N$, Shimizu T, Sugihara E, Oki $Y$, Sumikawa $Y$, et al. Regulation of MKL1 via actin cytoskeleton dynamics drives adipocyte differentiation. Nat Commun. 2014;5:3368.

33. Shaposhnikov D, Descot A, Schilling J, Posern G. Myocardin-related transcription factor $A$ regulates expression of Bok and Noxa and is involved in apoptotic signaling. Cell Cycle. 2012;11(1):141-50.

34. Milyavsky M, Shats I, Cholostoy A, Brosh R, Buganim Y, Weisz L, et al. Inactivation of myocardin and p16 during malignant transformation contributes to a differentiation defect. Cancer Cell. 2007:11(2):133-46.

35. Shaposhnikov D, Kuffer C, Storchova Z, Posern G. Myocardin related transcription factors are required for coordinated cell cycle progression. Cell Cycle. 2013;12(11):1762-72

36. Barisoni L, Mokrzycki M, Sablay L, Nagata M, Yamase H, Mundel P. Podocyte cell cycle regulation and proliferation in collapsing glomerulopathies. Kidney Int. 2000;58(1):137-43.

37. Wang S, Kim JH, Moon KC, Hong HK, Lee HS. Cell-cycle mechanisms involved in podocyte proliferation in cellular lesion of focal segmental glomerulosclerosis. Am J Kidney Dis. 2004;43(1):19-27.

38. Srivastava T, Garola RE, Whiting JM, Alon US. Cell-cycle regulatory proteins in podocyte cell in idiopathic nephrotic syndrome of childhood. Kidney Int. 2003;63(4):1374-81.

39. Shankland SJ, Floege J, Thomas SE, Nangaku M, Hugo C, Pippin J, et al. Cyclin kinase inhibitors are increased during experimental membranous nephropathy: potential role in limiting glomerular epithelial cell proliferation in vivo. Kidney Int. 1997;52(2):404-13. 
40. Hoshi S, Shu Y, Yoshida F, Inagaki T, Sonoda J, Watanabe T, et al. Podocyte injury promotes progressive nephropathy in zucker diabetic fatty rats. Lab Invest. 2002:82(1):25-35.

41. Nitta K, Horita S, Honda K, Uchida K, Watanabe T, Nihei H, et al. Glomerular expression of cell-cycle-regulatory proteins in human crescentic glomerulonephritis. Virchows Arch. 1999;435(4):422-7.

42. Xu H, Wu X, Qin H, Tian W, Chen J, Sun L, et al. Myocardin-Related Transcription Factor A Epigenetically Regulates Renal Fibrosis in Diabetic Nephropathy. J Am Soc Nephrol. 2014; [Epub ahead of print].

43. Fintha A, Gasparics Á, Fang L, Erdei Z, Hamar P, Mózes MM, et al Characterization and role of SCAl during renal fibrosis and epithelialto-mesenchymal transition. Am J Pathol. 2013;182(2):388-400.

44. Yang S, Du J, Wang Z, Yuan W, Qiao Y, Zhang M, et al. BMP-6 promotes E-cadherin expression through repressing deltaEF1 in breast cancer cells. BMC Cancer. 2007;7:211.

\section{Submit your next manuscript to BioMed Central and take full advantage of:}

- Convenient online submission

- Thorough peer review

- No space constraints or color figure charges

- Immediate publication on acceptance

- Inclusion in PubMed, CAS, Scopus and Google Scholar

- Research which is freely available for redistribution 